\title{
EL MUNICIPIO DE SAN BLAS Y LAS POLÍTICAS PARA EL DESARROLLO RURAL EN NAYARIT, MÉXICO
}

\section{THE SAN BLAS MUNICIPY AND THE RURAL DEVELOPMENT POLITICS IN NAYARIT, MÉXICO}

Francisca López Regalado*

\section{RESUMEN}

En las últimas décadas, las agencias financieras internacionales han destacado la importancia de las políticas públicas como dispositivo teórico y metodológico frente a la administración pública centralizada. En el discurso de estos organismos, la participación social y la descentralización son los ejes principales, mientras que desde una postura crítica, se aprecia como una forma de apropiación de las demandas de la sociedad para la preservación del poder de forma centralizada. En este marco, se analizan y discuten algunas modificaciones en el Estado mexicano como las reformas al art. 115 constitucional y la Ley para el Desarrollo Rural Sustentable (LDRS). Asimismo, esto se relaciona con los actores institucionales y de la ruralidad del municipio de San Blas, para reflexionar sobre las limitaciones y las posibilidades municipales en la definición de políticas para el desarrollo rural.

PALABRAS CLAVE: MÉXICO * POLÍTICA GUBERNAMENTAL * PLANIFICACIÓN RURAL * DESARROLLO RURAL * GOBERNABILIDAD

\section{ABSTRACT}

In recent decades, international financial agencies have stressed the importance of public policies as theoretical and methodological device against the governmental centralization. In the discourse of these agencies, social participation and decentralization are the main axes, whereas from a critical perspective, it is appreciated as a form of appropriation of social demands in order to preserve and centralize power. From the latter frame work, we analyze and discuss some changes in the Mexican state as reform stoart.115 of the Constitution and the Sustainable Rural Development Law (SRDL), analysis which lead us to institutional and rural actors from the municipality of San Blas, Nayarit, aspects and voices that, in the end, enable us to reflect about limitations and municipal possibilities in defining policies for rural development.

KEYWORDS: MEXICO * GOVERMENT POLICY * RURAL PLANNING * RURAL DEVELOPMENT * GOVERNANCE

Programa Académico de Ciencia Política de la Universidad Autónoma de Nayarit, México.

francis_lr@yahoo.com 


\section{DE LA ADMINISTRACIÓN A LAS POLÍTICAS} PÚBLICAS

En las últimas décadas, tanto en los organismos financieros internacionales, como en los espacios académicos y en diversas esferas de la gestión gubernamental del Estado mexicano, ha ocurrido no solo una modificación conceptual, sino una pretensión explícita de transformaciones en el diseño, instrumentación y evaluación de los cursos de acción. En este marco, las políticas públicas y la gobernanza adquieren relevancia frente a la administración pública. Difícilmente se podrían entender estos nuevos diseños institucionales al margen de los cambios en las relaciones de poder a escala mundial, planteando entre otras cosas, la reformulación de la relación entre las distintas esferas de gestión gubernamental como con los diversos sujetos del actuar institucional.

Vilas (2000) caracteriza a la actual etapa de globalización y de neoliberalismo e insiste en que no es un inicio, ni una fase en sí misma, sino la culminación de un ciclo de acumulación del capital que se abrió hace más de 70 años y en el presente, se ha desplegado en una modificación de las relaciones hegemónicas. Transformaciones que se relacionan con las estrategias de las empresas para contrarrestar sus dificultades para sostener los ritmos de acumulación. Así, han acelerado su transterritorialización mediante la descentralización, cuyas formas de competencia operan principalmente a través de la calidad, la diversificación y la flexibilidad.

Estos despliegues en redes conducen a un debilitamiento del carácter territorial del Estado nación cuando las estrategias empresariales requieren de la flexibilidad de los procesos productivos, de la elasticidad de las relaciones laborales, de una serie de ajustes en las directrices de la política económica y monetaria, así como en el marco regulatorio. Procesos que en su conjunto tienen como objetivo permitir la movilidad del capital transnacional en el territorio nacional.

En este contexto de demandas cruzadas del capital y de la sociedad, el Estado ha perdido legitimidad y con ello, se ha visto impelido a modificar su organización, funciones y su gestión. Las transformaciones conceptuales que acompañan este tránsito se hacen presentes. Se abandona el término de administración pública $y$ aparecen la gobernabilidad, la gobernanza $y$ las políticas públicas, resignificando con ello al federalismo, a los espacios locales y a la esfera de poder municipal.

En México, estas emergencias teóricas e instrumentales ocurrieron en un contexto de crisis y abandono del anterior modelo económico, expresado en la liberalización comercial, el adelgazamiento del Estado, las reformas políticas en el sistema electoral como en el de la función y gestión gubernamental. En esta última, se inscriben las reformas al art. 115 constitucional a partir del año 1983, marcando nuevas directrices y desafíos al gobierno municipal. De su carácter local y en consecuencia, de su cercanía con la comunidad, se desprende su principal función de atención a las necesidades comunitarias, de preservación del orden público o bien, de reconducción de las demandas sociales fuera de su espacio.

Si se reflexiona desde el contexto anteriormente señalado, se asocia con las posibilidades de desarrollo local y con la gobernabilidad. No es casual entonces, encontrar que en las propuestas teóricas, metodológicas e instrumentales, desde los diferentes enfoques, el municipio sea un espacio privilegiado y potencial para la política pública local.

De forma sintética, se enuncia que estas reformas se orientan a la redefinición de funciones para el logro de esa proximidad con la ciudadanía. La precisión de los servicios públicos objeto de su administración, las modificaciones en materia hacendaria para promover la autonomía financiera y fundamentalmente, el fortalecimiento del municipio como órgano de gobierno mediante el cuerpo colegiado del Ayuntamiento, facultándolo para el ejercicio de las funciones ejecutiva y legislativa.

Con las reformas al art. 115 Constitucional se sustituye el término "Administración" por el de "Gobierno", refrendando con ello la gestión y reivindicando el carácter político del municipio. Las implicaciones son múltiples; sin embargo, para efectos de este trabajo, interesa destacar las siguientes - relacionadas con el federalismo-: la modificación de las relaciones 
intergubernamentales asociadas a la descentralización de funciones y recursos; con ello la posibilidad de definición de políticas locales en el ámbito de su competencia y los vínculos de esos cambios con el desarrollo, particularmente el rural.

Sin reducirse a estos, los cursos de acción públicos como las estrategias descentralizadoras, se encuentran de inicio, orientados por la legislación respectiva y perfilados en los planes de desarrollo, vistos estos no solo desde los contenidos sino desde los procesos para su elaboración, logro y evaluación. Para el tema en estudio, ese marco jurídico se encuentra en la Ley de Desarrollo Rural Sustentable (LDRS), misma que se reseña en apartados posteriores. Ahora bien, se considera que estos objetivos $y$ cauces legales, guardan coherencia con las directrices globales arriba mencionadas $y$ sin que implique una reproducción mecánica, estas se relacionan con propuestas metodológicas, contenidos teóricos y con modos particulares de conocimiento. Por lo tanto, es pertinente debatir sobre los enfoques predominantes en políticas públicas y cuestionarse acerca de las concepciones y sus consecuencias sobre la participación social en los espacios municipales.

\section{PANORAMA GENERAL DE LAS PRINCIPALES TEORÍAS SOBRE POLÍTICAS PÚBLICAS}

\section{LAS POLÍTICAS PÚBLICAS Y ¿LA DESPOLITIZACIÓN?}

Aguilar (2000) ubica la emergencia de las políticas públicas como respuesta a las fisuras de la relación entre la sociedad y el Estado norteamericanos de los años 50. Desde el punto de vista de este autor, los quiebres cuestionan a la planificación centralizada y se fincan en uno de los supuestos centrales de la economía neoclásica: los recursos son limitados y escasos. Continúa afirmando que esta premisa en la gestión gubernamental, implica que cada decisión tiene costos de oportunidad que pueden ser onerosos y si a ello se le suma que en la gestión se omitió la elección racional, los resultados condujeron a la existencia de un Estado limitado fiscalmente. De ahí se afirma que son necesarias la privatización, la desregulación, la descentralización y la corresponsabilidad ciudadana.
En el plano teórico, estas exigencias de una visión instrumental de la gestión, pueden encontrarse en la estrategia de Laswell, citado en Aguilar (2000), quien centra su discusión en una distinción y una relación: la política $y$ del conocimiento de la política. Enseguida, identifica a la primera, con decisión democrática gubernamental, es decir, la gestión. El conocimiento de esta remite necesariamente a la ciencia; de ahí afirma que ciencia y política tendrían que estar integradas para afianzar una política sustentada en criterios de racionalidad. Los planteamientos de Laswell derivan en una gestión gubernamental despojada de la noción del poder como esencia de la politicidad del Estado.

En esta perspectiva, la gestión no se queda sola, tiene al conocimiento adjetivado de científico. En este espacio, persiste el debate alrededor de la relación entre gestión y política, cuando se distingue entre una ciencia sobre el proceso de la política y en la política. Se sostiene que el primero, desde la teoría positiva busca explicar cómo se determina la publicidad de ciertos asuntos y se excluye a otros. Mientras que el segundo, aporta métodos para que la selección y desarrollo de los cursos de acción, se sustenten en decisiones provenientes de la teoría normativa de carácter tecnológico. La distinción no quedó ahí, se definieron, dicotomizaron y se separaron los campos de estudio de la ciencia política y de las políticas públicas. Epistemológicamente, lo que está en juego es el pluralismo clásico fincado en la explicación racional, frente al comportamiento observable de los sujetos (behavioral revolution); es decir, los modelos hipotético deductivos basados en la maximización de utilidades, frente al empleo de técnicas e instrumentos para la selección de la mejor opción que reporte mayor eficiencia y utilidad. En ambos casos, la utilidad es el hilo conductor.

Desde esta última óptica instrumental, las políticas públicas son entendidas en sí mismas, toman de la ciencia el aporte de la aplicabilidad. Cuestionan no solo la validez universal de la administración centralizada, sino a la ciencia que indique el deber ser social. Sin embargo, en una pretensión de neutralidad 
valorativa, se sostiene que la ciencia sí puede contribuir a aclarar $y$ ordenar preferencias $y$ metas de los actores políticos, la licencia es concedida por la inclusión del carácter técnico, entonces, se puede hablar de valoración técnica, juicio técnico, tecnología e ingeniería social.

El desarrollo posterior $y$ principalmente, las discusiones recientes cuestionan el carácter instrumental de la administración pública y su separación de la política. Desde un ángulo de estudio de la ciencia política, Casar y Maldonado (2008) argumentan la insostenibilidad de un análisis aislado. Es en la formación de la agenda, en tanto proceso constitutivo de las políticas públicas, donde el vínculo es más evidente. Al discriminar alternativas, analizar la viabilidad e impacto de las mismas, se trasciende el plano empírico y remiten al carácter de los mecanismos de intermediación política. La formación de la agenda y la definición de problemas públicos son objeto de las políticas públicas. La manera en cómo se definen y construyen los problemas públicos y cómo se procesan y se toman las decisiones afirman es uno de los objetos de estudio fundamentales de la ciencia política.

Casar y Maldonado (2008) exponen los aportes de la ciencia política, en el sentido de que este proceso de formación de agenda acontece en un contexto de inviabilidad del pluralismo clásico. El argumento es la racionalidad limitada planteada entre otros autores por Keneth Arrow y Mancur Olson (1965).

Sin entrar en una exposición exhaustiva, interesa decir que se asientan en los siguientes argumentos: la agregación de preferencias individuales $y$ la búsqueda de la racionalidad no necesariamente se traducen en bienes colectivos; asimismo, la racionalidad se encuentra limitada por la insuficiencia de información. Condiciones que explican el tránsito del pluralismo clásico a esa Teoría de la Acción Colectiva fincada en la maximización de utilidad. La diferencia es que no se busca la alternativa óptima, sino una opción satisfactoria. Vergara (1993) sostiene el carácter cuestionador del neoinstitucionalismo frente a la racionalidad limitada, pues esta no tiene la suficiente capacidad explicativa acerca de lo que debería suceder — de acuerdo al modeloen relación con lo ocurrido realmente. De ahí, se explica que el neoinstitucionalismo precisa de modelos que expresen la incertidumbre.

En la exposición de Casar (2008), se señala que desde la perspectiva sistémica e institucional de las políticas públicas, se reconocen dos aspectos: las instituciones estatales se encuentran permeadas por la política y tienen la capacidad de moldear el comportamiento de los actores políticos. Destacan la importancia de este plano institucional y la necesidad de analizar el comportamiento de los individuos y su relación con los grupos a quienes están dirigidas las políticas, proponen analizar la variable institucional, la variable social y la variable internacional.

Recapitulando, la vertiente del planteamiento laswelliano orientado a las "policy making" sustituye las relaciones de poder por el conocimiento científico y bajo este se arroga la posibilidad de influir en los cursos de acción para la sociedad. Subyace un posicionamiento inductivo en el que mediante el comportamiento observable de las condiciones particulares y de la aplicación de técnicas sobre estas, se definen líneas de acción generales para el conjunto de la sociedad. En la otra línea, la del "policy process" se ubica el pluralismo clásico y la Teoría de la Acción Colectiva reseñada, cuyo punto de partida es que todos los actores se conducirán de forma racional para dar lugar a los modelos hipotéticos generales, caracterizados por la matematización del comportamiento social y la expectativa de una determinada conducta.

Lo anterior lleva a afirmar que - hasta lo aquí expuesto- el debate no ocurre entre posturas epistémicas diferentes al positivismo. Antes bien, la discusión gira entre un enfoque inductivo y deductivo o lo que es lo mismo, entre el empirismo y el neopositivismo.

En esa distinción, se destaca lo siguiente: mientras un enfoque no solo dicotomiza y separa la política de las políticas públicas; el otro, aunque asume su articulación, concibe a la política como ese procesamiento de las demandas ciudadanas en problemas públicos cuyo sustrato es la racionalidad maximizadora de 
ganancias, pero al no ocurrir en igualdad de representación, recurre al institucionalismo $y$ al neoinstitucionalismo como esa categoría básica de aproximación teórica y metodológica de estudio de la política e implícitamente del Estado. En esta óptica, este es una especie de árbitro cuyo sistema de sanciones y estímulos orienta la acción colectiva, dirime los conflictos sobre la distribución y procesa las demandas sociales, a través de los comportamientos estratégicos o de las preferencias congeladas. En esta acepción está latente esa noción de moldear la conducta social observable mediante un esquema de premios $y$ castigos. El enfoque inductivo y conductista se asoma en esta concepción del Estado.

Finalmente, en esta perspectiva sistémica e institucional se distingue a la agenda pública de la agenda política y de la gubernamental. La agenda pública se entiende como el conjunto de temas que la ciudadanía busca colocar; asimismo, mientras que la agenda política está constituida por el conjunto de temas que alcanzan prioridad en el debate y la acción de aquellos actores políticos que por su posición - recursos, facultades y poder - tienen capacidad para impulsarlas (Casar, 2008).

\section{EL DISCURSO, EL PODER Y LAS POLÍTICAS PÚBLICAS}

En un ángulo fenomenológico que se aleja de una visión de lo social como agregación de preferencias $y$ conjuntos (Edelman $y$ Schön, citados en Casar y Maldonado, 2008), las políticas públicas son abordadas desde una dimensión simbólica y enfatizan la primacía de los significados compartidos sobre la definición de los asuntos públicos porque en ellos subyacen percepciones de dominación. Al revelar estas apreciaciones se tiene una función emancipadora.

Con sus matices, aunque en esa misma perspectiva, Finnemore y Sikkink (citados en Cejudo, 2008), se afianzan en el constructivismo para el estudio de las políticas públicas. En este enfoque, es la intersubjetividad - los significados compartidos moldeados por factores ideacionales, no materiales, no reductibles a lo individual - la que construye los intereses de los actores. Las categorías de análisis son las ideas, normas $y$ argumentos compartidos que dan origen y explican la acción social, es decir, el discurso. El proceso de políticas, la definición de problemas, la formación de agenda pública y su evaluación, es en este enfoque, un juego para ganar y ejercer el poder, mediante discursos en competencia que reflejan la distribución del poder y la capacidad de los actores de imponer significados.

Paradójicamente, los mismos autores que refutan la visión instrumental y transitan a otras categorías de análisis de lo social, plantean las limitaciones del enfoque constructivista cuando afirman que no tiene una metodología precisa, al usar el análisis textual de contenidos o las comparaciones históricas y la observación participante. Como se observa, se podría estar confundiendo, el método con la técnica. Asimismo, se continúa ubicando las limitaciones en el criterio de validación, cuando sostienen que "al renegar del positivismo se pierde la posibilidad de tener estándares de validez" (Finnemore y Sikkink citados en Cejudo, 2008). Queda la impresión de cierta nostalgia de validar con categorías del positivismo, nociones que no guardan correspondencia con esta postura.

Un segundo límite es que el uso exclusivo de este enfoque explica poco y es necesario vincularlo con el entorno institucional. Supone al enfoque organizacional, al análisis político y al neoinstitucional. En otras palabras, se requiere de la combinación con otros enfoques para entender cómo el poder, los intereses y las instituciones modelan el discurso, y este a su vez, las políticas públicas.

Inicialmente, en este posicionamiento se presenta al discurso como esa categoría de aproximación a las políticas públicas, pues permite visibilizar $y$ analizar las percepciones que lo sostienen frente a discursos alternativos. Lo discutible es cuando plantean sus límites y dan marcha atrás, cuando en esa interpretación del discurso lo entienden en sí mismo y en el vacío, y no como síntesis a través de la cual se expresa lo político en los espacios institucionales y organizacionales. Parece continuar esa propuesta de análisis donde lo institucional se encuentra separado de la dimensión de lo político. 
LO PÚBLICO O LA ARTICULACIÓN ENTRE LO POLÍTICO Y LA POLÍTICA

Se advierte en Vilas (2000), una crítica al primer posicionamiento cuando enfatiza dos dimensiones básicas del Estado Nación: como expresión institucional de las relaciones de poder $y$ de principios de legitimación, es decir, su politicidad, $y$ la segunda, referida a las capacidades de gestión, que comprende a la administración pública o recientemente, a las políticas públicas. La segunda depende de la primera cuando en esa concepción, tanto la definición y ejecución de los cursos de acción como la asignación de recursos, se encuentran atravesados por su dimensión esencial: la politicidad.

Este eje básico del Estado se encuentra referido a las relaciones de poder institucionalizadas en el aparato Estatal, verbalizadas en el discurso, cristalizadas en el marco legal, desplegadas en las prácticas del sistema Estatal, llevando a otra forma de entendimiento de la gestión gubernamental. Sus caras y rumbos estarán atravesados por esa necesidad de legitimidad del poder. Alrededor de este propósito se encuentran otros posicionamientos críticos sobre la gobernabilidad democrática y la gobernanza, relacionados ambos, con las políticas públicas.

Diego y Rodríguez (2010) al hacer un recorrido por los términos anteriormente citados, señalan que en una de sus acepciones, la legitimidad es un atributo esencial e inherente al sistema político, le garantiza la estabilidad y la preservación del poder para la puesta en marcha de decisiones a través de los cauces institucionales. Precisamente, en esa necesidad de continuar centralizando el poder, se adjetiva de democrática a la gobernabilidad y se recurre a la gobernanza. Aunque en el primer término se apela a la política pública, la distribución del poder ocurre en los planos formales y tanto la desconcentración como la descentralización, no son más que estrategias de atención geográfica frente a un poder centralizado en sus funciones, profundizándose así la exclusión. Sin embargo, la política pública sí implicaría posibilidades autogestivas cuando se asocia a una radicalización de la democracia, ya que va más allá del espacio electoral y se encuentra necesariamente con la autonomía generando espacios de decisiones $y$ opciones alternativas de desarrollo. Dicho de otra forma, la política pública se relaciona con la gobernanza cuando se construyen los espacios autonómicos y participativos en concertación con los actores civiles, y cuando esta ciudadanía retoma funciones $y$ atribuciones usualmente en manos del espacio gubernamental.

Estas posibilidades autonómicas aunque no lo eluden, van más allá del espacio institucional y remiten a su conexión (o a la falta de esta) con esa matriz social y con sus relaciones de poder. De ahí se afirma que para el estudio de las políticas públicas, las formas específicas de aproximación a la dimensión de lo social, permiten un posicionamiento respecto de los enfoques reseñados.

En los enfoques fincados en la elección racional, aunque se busca la participación ciudadana, la sociedad civil es una dimensión externa a la política y al Estado. Las formas de socialidad son concebidas como suma, agregación de preferencias que desembocan en esa noción de la Acción Colectiva centrada en la maximización de ganancias. El principio metodológico es el individualismo. La consecuencia es la relación entre la política y la gestión, así como, la posibilidad de prospección de resultados. En esta perspectiva, las relaciones de poder son modelizadas, simplificadas y despojadas de su matriz social y sus significados. En otras palabras, los motivos y sentidos no tienen visibilidad en los espacios institucionales, predominando el planteamiento de que la política es actividad de determinados actores $y$ acontece en espacios específicos, principalmente, el institucional y en los canales de participación desde ahí definidos. No existe la reivindicación de lo político en esferas distintas a la institucional, en el conjunto de la vida social o en la llamada sociedad civil. Ahora bien, el neoinstitucionalismo afianzado en la rutinización de los sistemas institucionales, no plantea diferencias sustanciales sobre su forma de aproximación con lo social cuando señala la importancia de estudiarlo como una variable y cuando en esa noción de Estado subyace esa perspectiva conductista del castigo y la recompensa. Lo anterior tiene implicaciones en el análisis y la 
evaluación de las políticas; es decir, si el sustento teórico de las políticas públicas se asocia a la participación social y si lo social es un espacio de asimetrías que no son visibles desde la postura epistémica y teórica - desde la cual se construyen y diseñan los propósitos y cursos de acción- entonces, los resultados de los análisis, al fincarse en las mismas categorías analíticas con las que fueron construidas, puede dar lugar a indicadores o visiones parciales, que oculten o excluyan el sentido de lo político en la gestión.

Con respecto al ángulo fenomenológico, se consideran importantes algunos de sus posicionamientos: lo social y las relaciones de poder; estos pueden ser abordados desde la intersubjetividad como dimensión que muestra un campo de discursos en competencia para la imposición de significados. Esto es relevante porque descubre la multiplicidad de los sentidos que difícilmente pueden ser encerrados en esa racionalidad unívoca, negadora de las diferencias y centrada en la utilidad. Sin dejar de reconocer la importancia de la postura fenomenológica, surgen también algunos cuestionamientos, principalmente, cuando afirman la necesidad de incluir el análisis neoinstitucional y el análisis político, aparecen — cada unocomo dimensiones superpuestas, independientes entre sí, como si la dimensión política no atravesara a la institucional y como si estuviera ausente en los discursos. Por supuesto, no se trata de obviar estos últimos análisis (político e institucional), pero sí resulta discutible esta forma de aproximación cuando se intenta conjugar dos formas de conocer excluyentes, una fincada en la rutinización e identificación de variables y la otra, en las significaciones.

En la perspectiva crítica, las directrices de la administración pública o de las políticas públicas están marcadas por las relaciones de poder acuerpadas en el espacio institucional estatal. En esta correlación de fuerzas, el uso de este dispositivo teórico metodológico puede llevar a una preservación del poder centralizado o a una radicalización de los procesos democráticos. El rumbo se asocia a las condiciones sociopolíticas. Ahora bien, intentando articular los aportes al estudio de políticas públicas desde el ángulo fenomenológico y del crítico, el término "subjetidad" señalado por Echeverría (2001) es una contribución importante cuando lo destaca como una dimensión interna de lo físico y no metafísica, pertenece orgánicamente a la vida práctica y no es coexterna a ella. Desde esta categoría, sería cuestionable un análisis del discurso al margen de las prácticas y de la situación histórica.

De lo expuesto se desprende que no se trata de desechar al discurso en el estudio de las políticas públicas, por el contrario, se considera importante esta forma de aproximación, solo que es necesario interrogarse sobre, cómo en un juego de acción recíproca entre las condiciones materiales y el discurso, los intereses de los actores se visibilizan en el espacio institucional, se revelan en el marco legal, se verbalizan, se instrumentan con ciertas propuestas metodológicas y se despliegan en el quehacer de los actores a través de la coincidencia o de la tensión.

Por esta razón, es importante preguntarse por el discurso que sustenta las principales líneas metodológicas de la LDRS, búsquedas que remiten a las agencias financieras internacionales.

\section{LAS AGENCIAS FINANCIERAS INTERNACIONALES Y LOS RECIENTES APORTES METODOLÓGICOS PARA EL DESARROLLO RURAL}

Indagar sobre las políticas implica preguntarse por los cursos de acción, enunciados en principio, en el marco legal, en la planeación Estatal y en las estrategias metodológicas. En el desarrollo rural, estas disposiciones se relacionan con las principales categorías teórico metodológicas introducidas principalmente por el Banco Mundial (вм), el Banco Interamericano de Desarrollo (BID) y la Organización de las Naciones Unidas para la Alimentación (FAO). La propuesta se orienta a concebir una nueva ruralidad, cuyo criterio es la focalización en unidades territoriales en su diversidad y multidimensionalidad, que tiendan a las economías de escala, afianzadas en la participación y control social (Instituto Interamericano de Cooperación para la Agricultura-IIcA, Instituto Nacional para el Desarrollo de Capacidades del Sector Rural-INCA, 2010). 
Una noción central en esta metodología es el empoderamiento de los pobres y la descentralización mediante la formación de corredores económicos. Afirman que esas son condiciones para la reducción de la pobreza rural. Así, los criterios de cohesión territorial y social, desarrollo endógeno, tienen como sustrato los términos como redes $y$ capital social que se direccionan a la acción colectiva. No se trata de una política definida sectorialmente, sino a partir de territorios cuya especialización y competencia apuntan a una configuración regional. De ahí la importancia de las políticas públicas orientadas por la demanda, afianzándose los consejos territoriales como instancias de decisión y configuración de redes de políticas. La correspondencia con la perspectiva sistémica del primer enfoque analizado en el panorama teórico es evidente por los siguientes señalamientos: la gestión del desarrollo rural que abandona la visión sectorial para situarse en el carácter multidimensional del territorio está implícita en una interconexión de actividades, funciones, recursos y sujetos que trasciende el punto de vista centrado en lo estrictamente productivo. Si bien es cierto, se trata de una visión más integral y menos sectorial que busca respetar los valores culturales y las formas de participación social, el territorio es el objeto y sujeto de las políticas públicas, su cohesión social es el factor de atracción de inversión al ofrecer un ambiente de confianza y aunque aparentemente no se evade lo político, hay un propósito explícito de participación y control social que lleve a pactos y a mayor gobernabilidad. Por si alguna duda quedara, en esta misma línea, el Estado es entendido como proveedor de bienes públicos para posibilitar la construcción de la competitividad sistémica privada y para orientar las señales del mercado. El poder y lo político no son asumidos en este entendimiento del Estado.

En otros apartados se han señalado los cuestionamientos al enfoque sistémico, solo queda destacar que en esta propuesta, contradictoriamente a la apelación de la participación social, es el territorio el objeto y el sujeto de las políticas públicas. Aunque no se trata de desestimar la importancia de la dimensión territorial, se considera que esta adquiere sentido en relación con la significación, mundo de vida y estrategias de reproducción social de los sujetos; si bien, se toman en cuenta en esta propuesta, el territorio aparece en primer lugar, desplazando a los sujetos al segundo plano. En este deslizamiento, no se evade a lo político, pero sí se busca orientarlo al pacto $y$ a la gobernabilidad.

En los supuestos reseñados se finca el marco legal del federalismo para el desarrollo rural; la LDRs establece ese marco en el año 2001. Tiene como propósito una política rural integral mediante nuevas disposiciones cuyo propósito es el fomento de una nueva estrategia rural transversal. Es liderada por la Secretaría de Agricultura, Ganadería y Pesca (sagarpa) y busca promover la convergencia de políticas del sector rural mediante la creación de la Comisión Intersecretarial para el Desarrollo Rural Sustentable (cidrs) y del Consejo Mexicano para el Desarrollo Rural Sustentable (cmprs), cuya pretensión es la inclusión y representación de los intereses de los productores y agentes de la sociedad rural en su relación con los Consejos Municipal para el Desarrollo Rural Sustentable (cmprs) y los Consejos Distritales de Desarrollo Rural Sustentable (CDDRs).

En suma, si para las agencias financieras internacionales y para el Estado mexicano, el desarrollo rural se asocia al empoderamiento $y$ a la superación de la pobreza mediante la metodología fincada en el territorio, entendido este como un espacio de inclusión y convergencia de los intereses de los actores, es importante conocer si existe confluencia de estas directrices con los propósitos para el desarrollo rural en la esfera estatal.

\section{LAS POLÍTICAS ¿PÚBLICAS? Y LA PLANEACIÓN GUBERNAMENTAL EN NAYARIT}

LA PLANEACIÓN ESTATAL: 2005-2011 Y 2011-2017

En la planeación estatal correspondiente al sexenio 2005-2011, se explicita el enfoque de desarrollo regional y la conformación de mesorregiones, fincadas en la competitividad, sustentabilidad y encadenamiento productivo. En esa idea, la articulación productiva, de infraestructura, laboral, medioambiental, de salud, educativa, agraria, etc., muestran su 
correspondencia con los criterios marcados como importantes por las agencias financieras internacionales y la LDRs, aunque también se enfatiza la relevancia del criterio sectorial frente al regional.

Ahora bien, en concordancia con el lema "Gobierno de la Gente", central en sus estrategias de comunicación social y política, las políticas públicas y la nueva gestión se enuncian explícitamente en el discurso del Plan Estatal de Desarrollo 2011-2017. En este se destaca la participación ciudadana en los foros temáticos $y$ regionales, dando lugar a la definición de las siguientes políticas adjetivadas como públicas: Gobernabilidad, Calidad de Vida y Desarrollo Integral. En la primera, la seguridad, la legitimidad, la racionalidad, la eficiencia, la transparencia y la modernización parecieran ser sus vehículos. En la segunda, los aspectos centrales son la educación y la salud. Mientras que en la política para el desarrollo integral, se plantea de manera diferenciada una política para el campo centrada en la organización social, rentabilidad $y$ articulación productiva. Otra, para el desarrollo económico orientada al impulso de la construcción y dotación de infraestructura productiva $y$ finalmente, la política para el fomento de las actividades productivas cuyo propósito es la creación de un marco regulatorio eficiente y transparente para el impulso a pequeñas y medianas empresas.

Lo expuesto es relevante en varios sentidos: aunque se repiten algunos conceptos respecto del anterior sexenio estatal, se advierten oposiciones a la propuesta metodológica del Plan Nacional de Desarrollo correspondiente al desarrollo rural. En este sentido, la noción de territorialidad es el espacio de síntesis de la acción colectiva que tiene como propósito la competitividad y la articulación productiva. Presupone la conjugación del capital social con los recursos materiales, legales, ambientales, entre otros. Consecuentemente, existe una confluencia de políticas definidas territorialmente, de modo que cuando en esta planeación estatal se enuncia una política para el campo, otra para el desarrollo económico $y$ otra para las actividades productivas, queda desdibujada la noción territorial como síntesis de múltiples dimensiones.

Dicho de otra forma, cuando se define e instrumenta una política para el campo ¿no se incide en el mundo de vida campesino, en su economía y en sus actividades productivas? En consecuencia, pareciera existir cierta duplicidad, una visión que segmenta en lugar de la síntesis a través del territorio.

LA PLANEACIÓN EN EL MUNICIPIO DE SAN BLAS: 20052008, 2008-2011 Y 2011-2014

Si bien, se aprecia coherencia entre la planeación estatal 2005-2011 respecto de la federal, esas directrices se desdibujan en la planeación estatal 2011-2017, y tienden a desaparecer en los espacios municipales. En el trienio 2005-2008, se reconoce la importancia de la participación social y la convergencia de la sociedad civil en los Consejos de Desarrollo Municipal para lograr la modernización en la agricultura, la pesca y la ganadería.

Asimismo, la democracia, diversificación, sustentabilidad, integración productiva y comercial constituyen los ejes para el desarrollo rural. Mientras que en el periodo 2008-2011, estas líneas en la planeación, prácticamente se desvanecen $y$ aunque en algunos puntos coincide con los objetivos de la gestión anterior, la diferencia se encuentra en el cuerpo del documento. Este es una especie de monografía, cuyo contenido es una serie de datos estadísticos con escasa interpretación y conexión. Por su parte, la planeación de la actual gestión municipal 2011-2014, reconoce la importancia de la participación social. Para la formulación del plan, se realizó una serie de foros temáticos con la participación de la ciudadanía y de centros académicos $y$ de investigación social y tecnológica. Este ejercicio que resultó en una serie de propuestas muy concretas por ramas de actividad. Nuevamente, se utilizan términos de la nueva gestión pública; sin embargo, conceptualmente existe escasa articulación con las líneas metodológicas de la LDRS.

Recapitulando, se aprecia conexión conceptual y metodológica entre el primer enfoque expuesto sobre políticas públicas, la propuesta introducida por las agencias 
financieras internacionales y la LDRS, cuyas categorías son retomadas en la planeación estatal, particularmente en la gestión 2005-2011; mientras que en la gestión 2011-2017 existe continuidad conceptual cuando se definen políticas, aunque no parece tener el mismo sustento metodológico.

No obstante, esta metodología se desdibuja aún más en la esfera de gobierno municipal. Aunque existe un reconocimiento de la participación social y se definen políticas y estrategias amplias, estas no parten de un diagnóstico respecto de los ámbitos de competencias de la gestión del municipio.

Con lo expuesto, surgen algunas reflexiones acerca de los alcances y límites de las políticas públicas como dispositivo teórico y metodológico para la preservación del poder.

En principio, se advierte la relación entre los objetivos de la Ley para el Desarrollo Rural Sustentable, la metodología de las agencias financieras internacionales orientadas al desarrollo territorial y el modo de conocer que tiene como base a la Elección Racional y al Neoinstitucionalismo, cuya tendencia a la despolitización ha sido anteriormente señalada.

Asimismo, en el espacio objeto de este trabajo, no existe una reproducción discursiva lineal, automática y uniforme respecto de la apropiación e instrumentación de los objetivos, entre las esferas federal, estatal y municipal. En ese sentido y en la dimensión discursiva, las políticas públicas se encuentran limitadas como propuesta para la convergencia y la participación social.

Si se coincide con el posicionamiento de que en esta perspectiva, las políticas públicas fueron diseñadas con el propósito de la preservación del poder centralizado y si estas, no se despliegan teórica, metodológica e instrumentalmente en la misma dirección, es de suponerse entonces, que no cumplen o cumplen de manera parcial el objetivo para el que fueron creadas: la legitimidad. Con ello, no se infiere la presencia o ausencia de legitimidad, solo se enuncia que esta difícilmente se daría por estas vías, precisamente por la falta de apropiación de un discurso y quehacer institucional orientados a dar cauce a la participación social.
Ahora bien, las reflexiones anteriores se desprenden del análisis del marco legal y de la planeación de la administración pública en el recorte de realidad señalado, derivado de un análisis del discurso institucional. Sin embargo, en algunas críticas al ángulo fenomenológico, se cuestionaba un discurso deslocalizado y aislado de las condiciones materiales y de los intereses de los actores en cuestión. Al respecto, se considera importante comprender estas disposiciones en relación con las condiciones municipales para la instrumentación de las políticas públicas. En ese sentido, se concibe a los presupuestos en su origen, composición y orientación, como una expresión de la autonomía — no la única—o de la ausencia de esta.

\section{PRESUPUESTOS, DESARROLLO RURAL Y ¿AUTONOMÍA MUNICIPAL?}

Desde la perspectiva de Vilas, la autonomía es entendida como:

la capacidad de las autoridades públicas para definir objetivos $y$ fijar metas acordes a ellos, seleccionar y utilizar instrumentos eficaces $y$ eficientes, movilizar recursos en función de los objetivos y las metas, y mantener bajo control las restricciones dentro de las cuales operan las políticas públicas [...] En la medida en que el Estado es institucionalización de las relaciones de poder, los alcances efectivos y el sentido real de la autonomía Estatal derivan de las relaciones de poder así institucionalizadas (2000: 32).

Si bien, el autor se refiere a la autonomía del Estado en un sentido global, es evidente que la definición y logro de objetivos (abordados en la planeación) y la capacidad de movilización de recursos, son diferentes entre las esferas de la gestión gubernamental que lo conforman. Introducen el tema del federalismo y particularmente, el fiscal. Por lo tanto, la discusión sobre esas posibilidades de decisión para el desarrollo rural se encuentran asociadas a la distribución presupuestal. De acuerdo con esta, para el año 2012, solo el 12\% de los ingresos de la Municipalidad de San Blas son propios, por lo 
cual, existe una dependencia financiera del 88\% respecto del Estado y la Federación.

Ahora bien, si para el mismo ejercicio, solo el $37 \%$ se destina a inversiones y el resto a gasto corriente $y$ al pago de deuda, las posibilidades de movilización de recursos para el desarrollo rural dependen en buena medida del Fondo 3, particularmente del Fondo de Aportaciones para la Infraestructura Social Municipal (faIsM) ${ }^{1}$.

El farsm representa el $46 \%$ del total de aportaciones, orientándose tanto a la infraestructura productiva rural $y$ a la infraestructura de servicios básicos. Revisando las cantidades aplicadas en el ámbito rural, se muestra que para el ejercicio 2012, solo el 2,08\% del total de FAISM correspondió a la ejecución de un camino rural, el resto se orientó a empedrados, agua potable, reparación del sistema de bombeo, infraestructura básica educativa, mejoramiento de vivienda, gastos indirectos y desarrollo institucional. De lo anterior se desprende que los recursos ejercidos por el Municipio para el desarrollo rural son ínfimos.

Varias objeciones pudieran desprenderse de lo anteriormente señalado. Una de estas, es que el desarrollo rural no se reduce solo a actividades productivas y tiene un carácter integral que cruza una trama de aspectos que conforman el mundo de vida campesino, incluyendo

1 Los municipios perciben recursos federales por la vía de las Participaciones Fiscales Federales y de las Aportaciones denominadas como los Ramos 28 y 33. En el primer caso, se transfieren recursos a entidades federativas y municipios de acuerdo con la Ley de Coordinación Fiscal y los Convenios de Adhesión al Sistema de Coordinación Fiscal, la característica principal es que se trata de recursos de uso irrestricto, mientras que los recursos provenientes del Ramo 33 son de uso restringido. Los fondos que constituyen este Ramo son: Fondo para la Educación Básica y Normal (FAEB); Fondo de Aportaciones para los Servicios de Salud (FASSA); Fondo de Aportaciones para el Fortalecimiento de los Municipios y Demarcaciones Territoriales del Distrito Federal (FortamundF); Fondo de Aportaciones para la Educación Tecnológica y de Adultos (FAETA); Fondo de Aportaciones Múltiples (FAM); Fondo de Aportaciones para la Infraestructura Social Municipal (FAISM); Fondo de Aportaciones para la Seguridad Pública (FASP) y Fondo de Apoyo para el Fortalecimiento de las Entidades Federativas (FAFEF) (Pliego, 2010). los servicios básicos. Otro señalamiento pudiera ser que para las demandas cuya resolución está fuera de estos espacios, se fortalecen los Consejos Municipales de Desarrollo Rural Sustentable (cMDRs), entre otras cosas, para la reconducción mediante el vínculo con las instancias estatales $y$ federales a través de la concurrencia en los CDDRs y el cEDRs; es decir, el propósito de convergencia del Enfoque Territorial. Sin embargo, solo la actual administración enfatiza la necesidad de gestión y aunque en la planeación no se definen las estrategias para las relaciones intergubernamentales, existe una Dirección de Desarrollo Agropecuario orientada principalmente a la gestión.

En ese sentido, las posibilidades de definición de cursos de acción para el desarrollo rural, como es bien conocido, se encuentra atravesado por relaciones de poder y como se señaló, dependientes de la dimensión básica de la politicidad en tanto característica esencial del Estado (Vilas, 2000). No se trata de un Estado visto de forma monolítica frente a actores externos, sino de relaciones de poder, visibles en principio, entre las distintas esferas que componen a este, expresadas en el Municipio en esa doble sujeción al Estado y a la Federación. Las voces de los actores parecen confirmarlo.

\section{LA CONCURRENCIA EN LAS VOCES DE LOS ACTORES INSTITUCIONALES Y DE LA RURALIDAD MUNICIPAL Y LAS TENSIONES EN LOS CONSEJOS}

Sin duda, algunos puntos de la historia municipal y su dinámica económica, social y política son importantes para contextualizar las percepciones de los actores en el ámbito rural, por razones de espacio y alcance de este documento, solo se enuncian algunos referentes sobre la composición poblacional y productiva. De acuerdo a la siguiente información de la Secretaría de Desarrollo Social (sedesol): la población municipal es de 57461 habitantes, de la cual, el 53,3\% es urbana, concentrada en 4 localidades $^{2}$, mientras que el $46,7 \%$ está distribuida

2 Estas localidades son: San Blas (10 187 habitantes), Guadalupe Victoria (2 932), Mecatán (4 207) y Jalcocotán (2 657). 
en las 80 localidades restantes tipificadas como rurales ${ }^{3}$. También importa destacar que por sus $40 \mathrm{~km}$ de litoral y $25 \mathrm{~km}$ de esteros y ríos, hay una orientación hacia la pesca y al turismo. Lo expuesto se relaciona con la composición sectorial del Municipio: el 63\% de los ingresos provienen de los servicios, el $28 \%$ de las actividades primarias; $y$ el $9 \%$ de las actividades de transformación ${ }^{4}$ (INEGI, 2009).

Si bien, estas actividades se toman como referentes que ofrecen un panorama sobre las características generales de la población y sus actividades productivas, se parte de entender a la ruralidad como una forma de vida que en este contexto tendría que ver con las actividades reseñadas, pero tampoco podría ser reducida a estas, sino a este eje en relación con las significaciones de los actores $y$ a sus estrategias de reproducción social y cultural.

En las voces de los actores se encuentran tejidos el territorio, las actividades productivas y lo institucional, exponiendo un camino de dificultades para la convergencia y para la autonomía municipal. Por cuestiones de alcance, solo se presentan las de los actores orientados a las actividades más representativas en términos de los propósitos competitivos de la política rural.

Cuando se cuestionaba a un actor institucional municipal ${ }^{5}$ sobre la problemática rural en relación con el Municipio, sobresale el tono de protesta frente a otras esferas gubernamentales.

3 Los principales productos agrícolas son palma de coco, leucaena, melina, plátano, mango, aguacate, melón, jamaica, algodón, ciruela, pasto insurgente, pasto llanero, frijol, café, piña, arroz, guanábana, maíz, jitomate, sorgo y otros.

4 INEGI. Características principales de las Unidades Económicas del Sector Privado y Paraestatal que realizaron actividad durante el 2008.

5 Este informante de extracción priísta fue Presidente Municipal en la década de los 90 y ha ocupado diferentes cargos y funciones en diversas administraciones municipales y estatales. Actualmente, funge como Secretario de Desarrollo Agropecuario. Se considera que su información es relevante porque ofrece un panorama de los procesos de exclusión o concertación con los actores rurales, así como un referente importante sobre las dinámicas institucionales.
El 115 no se cumple, ;son puras políticas!... la libertad del municipio no es libre $[$ sic $] ..$. los recursos no se distribuyen de acuerdo a las necesidades, hay parcialidad. Antes eran otras las condiciones, un presidente municipal basificó a veintitrés, otro a cincuenta y seis, el Ayuntamiento tiene muchas cargas y deudas, de 103 millones, esto tenía que tronar...

Cuando yo fui presidente, me decía mi jefe, el gobernador: aquí van recursos que están etiquetados, si se desvían, me van a disculpar, yo los voy a tumbar de presidentes...el municipio tiene las manos amarradas y ahora más, con las deudas y tanta carga de trabajadores...

Sí, los Consejos Municipales de Desarrollo pueden ser una opción, se constituyeron para ver la problemática municipal $y$ al Distrito, se conforma con organizaciones económicas, civiles, tejidos, sociedad civil entre otros, se eligen representantes $y$ suplentes. Por lógica el presidente municipal es el presidente del Consejo, pero él no tiene mucho tiempo. Se hizo el análisis en mesas de trabajo. El principal problema es el mango, el frijol, el turismo, la pesca, el aguacate...los representantes no son propiamente los líderes...pero las organizaciones sí están representadas...

En el 2005 había un recurso, pero quienes eran los presidentes de los consejos (que era el presidente municipal) no los distribuían donde se necesitaban, ¿tú crees? Compraron machetes, palas y cosas de esas, eso hizo que ya no se asignaran recursos. Los Consejos no funcionan. Los representantes de las dependencias no le dan seguimiento a los acuerdos, el caso de los incendios forestales es un ejemplo. Después de una reunión que tuvimos, se dio aviso y no actuaron. Y todo pasa, nada sucede $y$ nos enemistamos. Ellos son los jefes, 
dependemos de ellos. ¿Qué hacemos? Luego en las reuniones no hay capacidad de decisión, mandan suplentes. El Consejo es un organismo autónomo que debe ser de observancia para todos. Pero si no entiende el gobierno en turno ¿qué es un Consejo? Entonces, jesto no sirve! Un ejemplo, mi General Zapata entregó la vida por la tierra, buscaba el reparto agrario, la producción, industrialización de productos, pero, pues sólo se cumplió lo de la repartición...

El gobierno nos utiliza con sus políticas públicas que no benefician a nadie... sobre quién define los programas, pues éstos ya están, no se pregunta qué necesita y qué quiere la gente. Pero de lo que hay, hemos gestionado recursos para un centro de acopio y un empaque, que beneficie a los productores de mango $y$ de aguacate, desde hace más de un año, pero nos dicen que no hay recurso. Gestionamos 44 proyectos por la vía de FAPPA Y PROMUSAG, ini uno salió! ¿Y tú crees que los campesinos saben hacer proyectos? Hay que pagar a quien los hace, afortunadamente los hizo un amigo y como no salieron, no se le pagaron, $y$ nosotros, hasta tenemos que poner dinero de nuestra bolsa, porque hay que hacer reuniones y ya ves cómo está el Ayuntamiento... Pero si se es afín al grupo que autoriza los recursos, entonces sí se les da a ciertos y cuales. A ellos sí se les da a conocer la normativa... son cuestiones políticas.

Si me preguntas cómo va esto del desarrollo rural, te digo que iva pésimo! (Entrevista, Anselmo Hernández, San Blas-Nayarit, 2013).

Lo expuesto llama la atención en varios sentidos: en principio, la crítica de un actor que ha sido y es parte del sistema que cuestiona, cuya propuesta pudiera responder más a esa sujeción del Municipio a las esferas federal $y$ estatal, que a la necesidad de inclusión de los actores rurales. Lo cierto es que desde su percepción se muestra que la eficacia de la gestión gubernamental no es una prioridad. Los cursos de acción se encuentran atravesados por esas lealtades a grupos políticos y el espacio municipal es percibido - en este caso- como un apéndice administrativo de otras esferas gubernamentales y no como un órgano de gobierno. Significaciones que no se encuentran en el vacío, por lo contrario, los juicios aquí vertidos están atados a las prácticas, donde el vacío institucional de la esfera estatal y federal se deja ver. También es visible la percepción sobre una larga serie de demandas irresueltas $y$ los cacicazgos en las mismas instancias municipales en diferentes administraciones. Evidentemente, no existe la participación social en la definición de cursos de acción para el desarrollo rural, mucho menos, seguimiento $y$ evaluación. Las políticas públicas se quedan en el nivel discursivo.

Lo anteriormente señalado es reforzado en los puntos centrales por los productores de aguacate, organización que aglutina a 1800 campesinos de los municipios de San Blas, Tepic y Xalisco ${ }^{6}$; asimismo, los campesinos productores de mango coinciden en esto. En la visión de uno de ellos, la problemática es la siguiente:

- Nosotros no nos dedicamos al puro mango, no vivimos de esto. En este producto, el principal problema es la comercialización, es un producto perecedero y el problema es la saturación del mercado, hace que baje el precio. La única salida es el procesamiento, darle valor agregado, porque ya vimos que los mismos compradores de siempre son los que se llevan la mayor parte de la ganancia... resulta que vino un comprador $y$ nos dice: $y$ a nos pusimos de acuerdo Layín ${ }^{7}$ y yo para no pagar más de quince mil pesos la hectárea. Y entonces le contesté: ah caray, no sabía que podían decidir sobre cosas de mi propiedad... (risas) ¿y también traes

6 Los testimonios de estos forman parte de una investigación en proceso, por lo extenso de su información, no se incluyen.

$7 \quad$ Esta persona fue presidente municipal en el trienio pasado. 
los espejitos?... porque inomás eso falta! Ellos acopian en toda esta región, él, (Layín) dicen que bajó recursos y con esos puso su empaque. Si nosotros vendemos a un precio, no el mejor, pero razonable para como están las cosas, vendemos en un promedio de un peso por kilo de mango, ya sea Tommy, Kent, Hayden o Ataulfo. Pero, si les vendemos a ellos, el precio anda como en cincuenta o sesenta centavos por kilo. ¿Qué nos hace venderles a ellos? La saturación de tempranillas, intermedias y tardías, todo eso es por el cambio climático, entonces, se desploma el precio... ¿qué más se necesita? Apoyos por supuesto, para procesar, pero éstos se dan a los más allegados, a los que tienen conexión. Los Consejos... no funcionan (Entrevista, C. P. G., San Blas-Nayarit, 2013) ${ }^{8}$.

Los cacicazgos incrustados en el poder local y las insuficiencias operativas de una política rural, muestran la desprotección del campesino que se ve arrojado a un escenario competitivo, echando mano de recursos propios para la producción, porque en buena medida invierte su trabajo sin la retribución justa; esfuerzos insuficientes para superar los escollos de la comercialización. Ahora bien, estos testimonios no muestran el mundo de vida campesino ni en este, la aleatoriedad de su ingreso, por ejemplo. Condiciones que cuestionarían la falta de integralidad en la definición de los programas gubernamentales que dan cuerpo a la política rural.

El caso de los campesinos ganaderos es similar; sin embargo, por el tipo de información $y$ las formas específicas de tramas e instancias para el ejercicio del control político y económico regional, se considera conveniente realizarlo en posteriores investigaciones. Solo resulta importante destacar que en esta actividad, las formas de control están asociadas a las mismas uniones ganaderas $y$ en toda la red tejida alrededor de la autorización de la compra y venta de ganado.

8 Se omite el nombre completo por motivos profesionales.

\section{CONCLUSIONES}

Aunque no se trata de una reproducción automática, uniforme y lineal, existen relaciones entre un modo de conocimiento de las políticas públicas, la propuesta metodológica de las agencias financieras internacionales y el marco legal para el desarrollo rural nacional. Este enfoque predominante sobre las políticas públicas se finca en la Elección Racional y en la Acción Colectiva. En la primera y en esta concepción de la segunda relacionada con la utilidad maximizadora de ganancias, existe una tendencia despolitizadora. Con sus matices y con diferentes intensidades, estas líneas permean la enunciación de los cursos de acción de la gestión de las distintas esferas gubernamentales: la federal, la estatal y la municipal. Sin embargo, como ha sido expuesto, este enfoque predominante se desdibuja en la actual administración estatal y principalmente, en el espacio municipal. En este espacio, en oposición al objetivo para el que fueron diseñadas, las políticas aparecen más como un proceso centralizador que un espacio para la participación social. La planeación para el desarrollo rural, sus propósitos y metas, se muestran más como un ejercicio retórico que como un proceso incluyente, orientador de las acciones gubernamentales $y$ los medios para su logro $y$ su evaluación. No se parte de un reconocimiento del origen, destino y alcance presupuestal. Ha sido manifiesto también, que no existe participación social en el seguimiento y en la evaluación.

La profundización en las razones de esta apropiación discursiva desigual escapa al objeto del presente artículo; sin embargo, es posible entenderlo como un proceso complejo, cuya comprensión lleva a diversas líneas actuantes entre sí. Se considera que una de estas se relaciona con el multicitado espacio institucional, con la falta de correspondencia entre los objetivos enunciados en la LDRS - cuyo propósito de convergencia implica al federalismo en los espacios rurales-, y el origen y distribución de recursos. En otras palabras, los objetivos del marco legal obedecen más a las consignas de las agencias financieras internacionales que a una voluntad real de modificación de prácticas para que estas tiendan a la descentralización gubernativa, administrativa y financiera hacia 
las instancias municipales. En esta esfera, aunque el marco legal reafirma la función de gobierno y la autonomía municipal, el origen $y$ composición de recursos parece contradecirla. Esto lleva a una percepción del espacio municipal como un apéndice administrativo de las instancias estatales $y$ federales, con espacios acotados en las estructuras institucionales creadas para la convergencia.

De igual forma, se piensa que la falta de resonancia en la práctica del marco legal $y$ del andamiaje institucional (CDRS, CDDRS, CEDRS $y$ CMDR) no se pudiera comprender al margen de la dimensión cultural e histórica. Dicho de otro modo, una propuesta descentralizadora no implica solo la definición de objetivos, recursos legales, económicos e instrumentales. Por el contrario, se encuentra atravesada por esas identidades, valores y disposiciones que modelan la vida social en su cotidianidad y desde una perspectiva histórica. De ahí surge la cuestión de cómo entender una propuesta que promueve la participación social desde arriba, cuando esta se ejerce $y$ se inserta en una añeja red de relaciones verticales $y$ clientelares producto de la historia de México y de Nayarit, historia que no es posible ampliar en este espacio, pero sí se desprende la necesidad de profundizar en ambas líneas.

Para efectos de este trabajo, se destaca la voz de los actores del desarrollo rural y su percepción sobre la exclusión ${ }^{9}$. Institucionales o no, en sus apreciaciones y sentires, la relación con las instancias municipales, estatales y federales no ha dado lugar a esos espacios participativos, ni ha implicado la toma de decisiones $y$ atribuciones. Estos continúan en el espacio gubernamental, principalmente en el federal y el estatal.

$9 \quad$ Varias de las reflexiones en el presente trabajo se desprenden de los testimonios de informantes clave. Por la forma de aproximación que no se ubica en una perspectiva instrumental, no se parte de una acumulación de encuestas, sino que busca partir de las voces de los campesinos y de los actores institucionales, cuyo quehacer, sentires y percepciones ofrecen un panorama general de la relación de los actores rurales con los institucionales en el Municipio de San Blas. Por supuesto, dan lugar a reflexiones sobre la ruralidad y las políticas públicas que no se agotan en el presente documento.
Por lo pronto, este enfoque predominante aparece en las planeaciones, más como un requisito formal, sin existir una apropiación por los actores institucionales en sus diferentes niveles ${ }^{10}$ $y$ por las afirmaciones anteriormente analizadas, se sostiene que no se trata solo de un problema de la eficiencia de la gestión, sino de la trama de poder que influye en los rumbos que toma la gestión gubernamental. Se cuestiona con esto a las propuestas teórico-metodológicas que abordan a la gestión pública en sí misma, como un aspecto solamente instrumental y se reafirma la importancia del reconocimiento del poder $y$ de lo político en las iniciativas de participación social. Ambos ejes contenidos en los ángulos fenomenológico y crítico de las políticas públicas, alrededor de los cuales resulta pertinente profundizar en su modo de conocer, en sus propuestas teóricas y en estrategias metodológicas y de instrumentación.

\section{BIBLIOGRAFÍA}

\section{LIBROS}

Aguilar Villanueva, Luis F. Problemas públicos y agenda de gobierno. México: Miguel Ángel Porrúa, 1996.

Aguilar Villanueva, Luis F. El Estudio de las políticas públicas. México: Miguel Ángel Porrúa, 2000.

Aguilar Villanueva, Luis F. La hechura de las políticas públicas. México: Miguel Ángel Porrúa, 2003.

Casar, María Amparo y Maldonado, Claudia. Formación de agenda y procesos de toma de decisiones: una aproximación desde la ciencia politica”. México: Centro de Investigación y Docencia Económicas-cIDE, 2008.

Cejudo, Guillermo M. Discurso y políticas públicas. Un enfoque constructivista. México: Centro de Investigación y Docencia Económicas-cIDE, 2008.

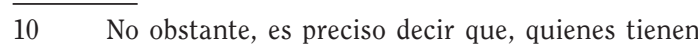
mayor contacto con los actores campesinos y su problemática, son los que aprecian las insuficiencias y perciben a la gestión gubernamental como un proceso centralizador en el que el Municipio se encuentra sujeto tanto al estado como a la Federación. 
Colomer, Joseph M. El enfoque de la elección racional en política. Madrid, España: Universidad Autónoma de Barcelona, 1991.

Diego, Roberto y Rodríguez, Carlos. Gobernabilidad y autonomía. Una crítica desde los enfoques participativos del desarrollo. México: Universidad Autónoma Metropolitana-uam, 2010.

Echeverría, Bolívar. "Ensayos Políticos". Pensamiento político ecuatoriano. Colección dirigida por Fernando Tinajero. Quito, Ecuador, 2011.

Gobierno del Estado de Nayarit. Ley de Ingresos para la Municipalidad de San Blas para el Ejercicio Fiscal 2012. México, 2011.

Gobierno del Estado de Nayarit. Plan Estatal de Desarrollo 2005-2011. México, 2005.

Gobierno del Estado de Nayarit. Plan Estatal de Desarrollo 2011-2017. México, 2012

Instituto Interamericano de Cooperación para la Agricultura-IIca y el Instituto Nacional para el Desarrollo de Capacidades del Sector Rural-inca. Hacia una gestión territorial: institucionalidad y concurrencia en la operación de los Consejos Municipales de desarrollo rural. Red para la gestión territorial del desarrollo rural en México. México: Instituto Interamericano de Cooperación para la Agricultura-IICA, 2010.

Instituto Nacional de Estadística y Geografía de México-INEG. Censos Económicos 2009. México: INEG, 2009.

Lahera Parada, Eugenio. Introducción a las políticas públicas. Chile: Fondo de Cultura Económica-fce, 2002.

Mardones, José María. Filosofía de las ciencia sociales y humanas. España: Anthropos Editorial, 1991.

Morris, P. Fiorina. "El método de la Teoría Positiva". Los modelos formales en ciencia politica. Madrid, España: Instituto de Estudios Fiscales, 1991.

Pliego, Iván. El federalismo fiscal en México: entre la economía y la política. México: Cámara de Diputados ux Legislatura, 2010.

Roemer, Andrés. La perspectiva de la elección pública. México: Instituto Tecnológico Autónomo de México-ITAM, 2010.
Sistema de Apoyo para la Planeación. Catálogo de localidades. México: Secretaria de Desarrollo Social-sedesol, 2013.

Suprema Corte de Justicia de la Nación-SCJN, México. Reformas constitucionales por artículo. Constitución Politica de los Estados Unidos mexicanos que reforma la del 5 de Febrero de 1857. México: scin, 2012.

Vergara, Rodolfo. Decisiones, organizaciones y nuevo institucionalismo. México: Universidad Autónoma MetropolitanaUAM, 1993.

\section{PUBLICACIONES PERIÓDICAS}

Olson, Mancur. "The logic of collective action; public goods and the theory of groups". Harvard Economic Studies 124. USA: Harvard University Press, 1965.

Vilas, Carlos M.. "Estado y mercado en la globalización: la reformulación de las relaciones entre política y economía. instituto". Revista de Sociología e Política 14. Brasil, Universidade Federal do Paraná. Junio 2000: 29-49.

\section{TEXTOS ELECTRÓNICOS}

Castells, Manuel. ¿Hacia el Estado Red? Globalización económica e instituciones políticas en la era de la información. En: $<$ http://www.trueque-marysierras.org. ar/biblioteca2.htm> [Consultado el 18 de enero de 2013].

\section{ENTREVISTAS}

Anselmo Hernández Sojo. Secretario de Desarrollo Agropecuario del xxxix Ayuntamiento de San Blas, Nayarit, México. Entrevista realizada en San Blas, Nayarit el día 18 de setiembre de 2013. Entrevistadora: Francisca López Regalado.

C.P.G. Entrevista realizada en San Blas, Nayarit el día 5 de agosto de 2013. Entrevistadora: Francisca López Regalado.

Fecha de ingreso: 01/04/2014 Fecha de aprobación: 14/07/2014 